\title{
A quantitative discounted central limit theorem using the Fourier metric
}

\author{
Guy Katriel \\ Department of Mathematics, ORT Braude College, \\ Karmiel, Israel
}

\begin{abstract}
The discounted central limit theorem concerns the convergence of an infinite discounted sum of i.i.d. random variables to normality as the discount factor approaches 1 . We show that, using the Fourier metric on probability distributions, one can obtain the discounted central limit theorem, as well as a quantitative version of it, in a simple and natural way, and under weak assumptions.
\end{abstract}

\section{Introduction}

Let $X_{n}(n \geq 0)$ be a sequence of i.i.d. real-valued random variables, with

$$
\mu=E\left(X_{0}\right), \quad \sigma^{2}=\operatorname{Var}\left(X_{0}\right)<\infty .
$$

For $a \in[0,1)$, we define the random variable

$$
S_{a}=\sum_{n=0}^{\infty} a^{n} X_{n} .
$$

Standard results ensure that (2) converges almost surely (see e.g. 2], Sec. 5.3). $S_{a}$ can be understood as the present value of a future stream of i.i.d. payments, where $a$ is the discount factor.

Gerber [6] proved, assuming that $X_{n}$ have finite third moments, that as $a \rightarrow 1$, the distribution of $S_{a}$ approaches a normal distribution: normalizing $S_{a}$ by setting

$$
\hat{S}_{a}=\frac{S_{a}-E\left(S_{a}\right)}{\sqrt{\operatorname{Var}\left(S_{a}\right)}}=\frac{\sqrt{1-a^{2}}}{\sigma} \cdot\left(S_{a}-\frac{\mu}{1-a}\right),
$$

we have

$$
\hat{S}_{a} \stackrel{D}{\rightarrow} N(0,1) \quad \text { as } a \rightarrow 1-
$$


that is, defining the corresponding cumulative distribution functions

$$
\begin{gathered}
F_{a}(x)=P\left(\hat{S}_{a} \leq x\right), \\
\Phi(x)=P(N(0,1) \leq x)=\frac{1}{\sqrt{2 \pi}} \int_{-\infty}^{x} e^{-\frac{u^{2}}{2}} d u,
\end{gathered}
$$

we have, for all $x \in \mathbb{R}$,

$$
\lim _{a \rightarrow 1-} F_{a}(x)=\Phi(x) .
$$

This is the discounted central limit theorem. Gerber also gave a quantitative bound of Berry-Eseen type for this convergence:

$$
\sup _{x \in \mathbb{R}}\left|F_{a}(x)-\Phi(x)\right| \leq C \cdot \frac{E\left(\left|X_{0}-\mu\right|^{3}\right)}{\sigma^{3}} \cdot(1-a)^{\frac{1}{2}},
$$

and one can take $C=5.4$ (we note that the formulation given in [6] is slightly different, but equivalent, because of the different normalization taken there). Subsequent works extended and refined the results of [6] in several directions (see e.g. [3, 8, 9, 10]).

Here we will prove a discounted central limit theorem without any assumption on moments higher than 2 , and also give a new and different quantitative bound for the convergence, in the case that some moment of order $s=2+\epsilon$ $(\epsilon>0)$ exists. This bound will be given in terms of a Fourier-based metric, which will be seen to provide a simple and natural approach to the study of discounted sums. A key observation underlying our proofs is that the distibution $F_{a}$ can be realized as a fixed point of a mapping on a space of distributions, which is a contraction with respect to this metric. Fourierbased metrics were introduced in connection with study of the Boltzmann equation [5], and have since found many applications (see [1] for a review). In particular in [7] these metrics have been used to obtain Berry-Esseen type inequalities.

We recall the definition of the Fourier-based metrics. For any real $s>0$, we denote by $\mathcal{P}^{s}$ the set of all distribution functions $G$ on $\mathbb{R}$ with finite moment of order $s$, and with expectation 0 and variance 1 :

$$
\begin{gathered}
\int_{-\infty}^{\infty}|x|^{s} d G(x)<\infty \\
\int_{-\infty}^{\infty} x d G(x)=0, \quad \int_{-\infty}^{\infty} x^{2} d G(x)=1 .
\end{gathered}
$$

To each distribution function $G$ we associate its characteristic function

$$
C_{G}(\xi)=\int_{-\infty}^{\infty} e^{-i \xi x} d G(x)
$$


If $s>0$, and $G, H$ are probability distributions, their Fourier distance of type $s$ is defined by

$$
d_{s}(G, H)=\sup _{\xi \neq 0} \frac{\left|C_{G}(\xi)-C_{H}(\xi)\right|}{|\xi|^{s}} .
$$

If $s \in[2,3]$ and $G, H \in \mathcal{P}^{s}$, then $d_{s}(G, H)<\infty$ (see [1, Proposition 2.6).

We prove that

Theorem 1. If (1) holds, then

$$
\lim _{a \rightarrow 1-} d_{2}\left(F_{a}, \Phi\right)=0 .
$$

(12) implies pointwise convergence of $C_{F_{a}}$ to $C_{\Phi}$, which, by Levy's Continuity Theorem (see e.g. [2], Sec 6.3), implies (7). The validity of the discounted central limit theorem (44), without any assumption on moments higher than 2, thus follows from Theorem 1- we note however that it also follows from previous results such as those in [3].

A quantitative version of Theorem 1 can be obtained if we assume that $X_{n}$ have a finite $s$-moment for some $s>2$. Set

$$
\hat{X}_{0}=\sigma^{-1}\left(X_{0}-\mu\right),
$$

and let $F$ denote its distribution function:

$$
F(x)=P\left(\hat{X}_{0} \leq x\right) .
$$

Note that by (1) have $F \in \mathcal{P}^{2}$.

Theorem 2. Assume $F \in \mathcal{P}^{s}$ where $s \in(2,3]$. Then, for $a \in(0,1)$

$$
d_{2}\left(F_{a}, \Phi\right) \leq\left[\frac{(s-2)\left(1-a^{2}\right)}{e \cdot a^{2}}\right]^{\frac{1}{2}(s-2)} \cdot d_{s}(F, \Phi) .
$$

Note that $s>2$ implies that the right-hand side of (14) goes to 0 as $a \rightarrow 1$, so that (14) implies (12) for $s>2$ (but not for $s=2$, which is the reason that Theorem 1 needs a separate proof).

Comparing the bound of Theorem 2 with Gerber's bound (8), we note several differences.

(1) Theorem 2 provides a bound whenever some moment of order $s>2$ is finite, while (8) requires a finite third central moment for $X_{0}$.

(2) The bound (8) is universal, hence does not take into account the distance between the distribution of $X_{0}$ and the normal distribution. In (14), the bound becomes small if $X_{0}$ is close to normal. 
(3) A major difference is of course the fact that the distance between distributions is measured differently: while (14) uses a Fourier metric, (8) uses the Kolmogorov metric. In fact it is possible to bound the Kolmogorov metric in terms of the $d_{2}$ metric: using the Berry-Eseen inequality (see [4], Sec. XVI.4, Lemma 2) we get

$$
\begin{gathered}
\left|F_{a}(x)-\Phi(x)\right| \leq \frac{1}{\pi} \int_{-T}^{T} \frac{\left|C_{F_{a}}(\xi)-C_{\Phi}(\xi)\right|}{|\xi|^{2}}|\xi| d \xi+\frac{24}{\pi T} \\
\leq \frac{1}{\pi} \cdot d_{2}\left(F_{a}, \Phi\right) \int_{-T}^{T}|\xi| d \xi+\frac{24}{\pi T}=\frac{1}{\pi} \cdot d_{2}\left(F_{a}, \Phi\right) T^{2}+\frac{24}{\pi T},
\end{gathered}
$$

and optimizing over $T$ gives

$$
\sup _{x \in \mathbb{R}}\left|F_{a}(x)-\Phi(x)\right| \leq \frac{3 \cdot 12^{\frac{2}{3}}}{\pi} \cdot\left(d_{2}\left(F_{a}, \Phi\right)\right)^{\frac{1}{3}},
$$

so that convergence in the $d_{2}$ metric implies convergence in the Kolmogorov metric (as well as in the Wasserstein metric, see [1], Theorem 2.21). However, it should be noted that using this bound together with (14) gives a bound of order $O\left((1-a)^{\frac{1}{6}(s-2)}\right)$ as $a \rightarrow 1$ for the convergence of the Kolmogorov metric, which in the case $s=3$ (which is relevant for this comparison) gives $O\left((1-a)^{\frac{1}{6}}\right)$, a weaker convergence rate than the one given by (8).

We thus conclude that none of the inequalities (8) and (14) is a consequence of the other, and each has its advantages. It might be an interesting problem to obtain bounds which combine the advanatges of the two inequalities.

\section{Proofs of the theorems}

Noting that $\hat{S}_{a}$ does not change if a linear function is applied to all $X_{n}$ 's, there is no loss of generality in proving our results under the normalization

$$
E\left(X_{n}\right)=0, \quad \operatorname{Var}\left(X_{n}\right)=1,
$$

which we will henceforth assume. Under this assumption the distribution $F$ given by (13) is simply the distribution of the $X_{n}$ 's:

$$
F(x)=P\left(X_{0} \leq x\right),
$$

and (3) becomes

$$
\hat{S}_{a}=\sqrt{1-a^{2}} \cdot S_{a}
$$

For $a \in[0,1)$, we now define the operator $T_{a}: \mathcal{P}^{2} \rightarrow \mathcal{P}^{2}$, whose unique fixed point will later be shown to be the distribution function $F_{a}$ of $\hat{S}_{a}$. If $G \in \mathcal{P}^{2}$, 
let $Y$ be a random variable with distribution function $G$. Let $X$ be a random variable independent of $Y$, with distribution function $F$ given by (15). Then $T_{a}[G]$ is defined to be the distribution function of $a Y+\sqrt{1-a^{2}} \cdot X$ :

$$
T_{a}[G](x)=P\left(a Y+\sqrt{1-a^{2}} \cdot X \leq x\right) .
$$

Since

$$
E\left(a Y+\sqrt{1-a^{2}} \cdot X\right)=a E(Y)+\sqrt{1-a^{2}} \cdot E(X)=0
$$

$\operatorname{Var}\left(a Y+\sqrt{1-a^{2}} \cdot X\right)=a^{2} \operatorname{Var}(Y)+\left(1-a^{2}\right) \operatorname{Var}(X)=a^{2}+\left(1-a^{2}\right)=1$,

we indeed have $T_{a}[G] \in \mathcal{P}^{2}$.

By the above definition, the $n$-fold composition $T_{a}^{n}[G](n \geq 1)$ is the distribution function of the random variable $Y_{n}$ given by the autoregressive process

$$
Y_{n+1}=a Y_{n}+\sqrt{1-a^{2}} \cdot X_{n}, \quad n \geq 0
$$

when $G$ is the distribution function of $Y_{0}$.

In terms of characteristic functions we have

$$
C_{T_{a}[G]}(\xi)=C_{F}\left(\sqrt{1-a^{2}} \cdot \xi\right) \cdot C_{G}(a \xi) .
$$

The following Lemma summarizes properties of the operator $T_{a}$.

Lemma 1. For $a \in[0,1)$ :

(i) If $G, H \in \mathcal{P}^{2}$ then For any integer $n \geq 1$

$$
d_{2}\left(T_{a}^{n}[G], T_{a}^{n}[H]\right) \leq a^{2 n} \cdot d_{2}(G, H) .
$$

(ii) $F_{a}$, given by (5), is a fixed point of $T_{a}$, and if $a>0$ it is the unique fixed point.

(iii) For any $G \in \mathcal{P}^{2}$ we have

$$
d_{2}\left(G, F_{a}\right) \leq \frac{d_{2}\left(G, T_{a}[G]\right)}{1-a^{2}}
$$

Proof. (i) For $n=1$ we have, using (18) and the fact that $\left|C_{F}(\xi)\right| \leq 1$,

$$
\begin{gathered}
d_{2}\left(T_{a}[G], T_{a}[H]\right)=\sup _{\xi \neq 0} \frac{\left|C_{F}\left(\sqrt{1-a^{2}} \cdot \xi\right)\right| \cdot\left|C_{G}(a \xi)-C_{H}(a \xi)\right|}{\xi^{2}} \\
\leq \sup _{\xi \neq 0} \frac{\left|C_{G}(a \xi)-C_{H}(a \xi)\right|}{\xi^{2}}=a^{2} \cdot \sup _{\xi \neq 0} \frac{\left|C_{G}(a \xi)-C_{H}(a \xi)\right|}{(a \xi)^{2}}=a^{2} \cdot d_{2}(G, H) .
\end{gathered}
$$


Proceeding by induction, we have

$$
d_{2}\left(T_{a}^{n+1}[G], T_{a}^{n+1}[H]\right) \leq a^{2} \cdot d_{2}\left(T_{a}^{n}[G], T_{a}^{n}[H]\right)=a^{2(n+1)} \cdot d_{2}(G, H) .
$$

(ii) We denote equality in distribution of two random variables by $\stackrel{D}{=}$. Let $X$ be a random variable with distribution $F$, independent of $S_{a}$. We claim that

$$
a \hat{S}_{a}+\sqrt{1-a^{2}} \cdot X \stackrel{D}{=} \hat{S}_{a}
$$

which implies $T_{a}\left[F_{a}\right]=F_{a}$. To show (21), note that we have

$$
S_{a}=\sum_{n=0}^{\infty} a^{n} X_{n}=X_{0}+a \sum_{n=0}^{\infty} a^{n} X_{n+1} \stackrel{D}{=} X+a S_{a},
$$

so that, using (16),

$$
a \hat{S}_{a}+\sqrt{1-a^{2}} \cdot X=\sqrt{1-a^{2}} \cdot\left[a S_{a}+X\right] \stackrel{D}{=} \sqrt{1-a^{2}} \cdot S_{a}=\hat{S}_{a},
$$

so we have (21).

To show uniqueness of the fixed point when $a>0$, assume $G \in \mathcal{P}^{2}, T_{a}[G]=$ G. Using (19),

$d_{2}\left(F_{a}, G\right)=d_{2}\left(T\left[F_{a}\right], T[G]\right) \leq a^{2} \cdot d_{2}\left(F_{a}, G\right) \Rightarrow d_{2}\left(F_{a}, G\right)=0 \Rightarrow G=F_{a}$.

(iii) By (19) we have

$$
d_{2}\left(T_{a}^{k}[G], T_{a}^{k+1}[G]\right) \leq a^{2 k} d_{2}\left(G, T_{a}[G]\right),
$$

so the triangle inequality gives

$$
d_{2}\left(G, T_{a}^{n}[G]\right) \leq \sum_{k=0}^{n-1} d_{2}\left(T_{a}^{k}[G], T_{a}^{k+1}[G]\right) \leq \frac{1-a^{2 n}}{1-a^{2}} \cdot d_{2}\left(G, T_{a}[G]\right)
$$

From (i),(ii) we have

$$
d_{2}\left(T_{a}^{n}[G], F_{a}\right)=d_{2}\left(T_{a}^{n}[G], T_{a}^{n}\left[F_{a}\right]\right) \leq a^{2 n} \cdot d_{2}\left(G, F_{a}\right) .
$$

Therefore, using the triangle inequality and (22),(23),

$$
\begin{aligned}
d_{2}\left(G, F_{a}\right) & \leq d_{2}\left(G, T_{a}^{n}[G]\right)+d_{2}\left(T_{a}^{n}[G], F_{a}\right) \\
& \leq \frac{1-a^{2 n}}{1-a^{2}} \cdot d_{2}\left(G, T_{a}[G]\right)+a^{2 n} \cdot d_{2}\left(G, F_{a}\right),
\end{aligned}
$$

and taking $n \rightarrow \infty$ we obtain (20).

The following Lemma plays a key role in proving the theorems: 
Lemma 2. Let $\Phi$ be the Normal distribution function (6). Then for a $\in$ $[0,1)$

$$
d_{2}\left(F_{a}, \Phi\right) \leq \sup _{w \neq 0}\left[e^{-\frac{(a w)^{2}}{2\left(1-a^{2}\right)}} \cdot \frac{\left|C_{F}(w)-C_{\Phi}(w)\right|}{w^{2}}\right]
$$

Proof. Applying (20) with $G=\Phi$ we have

$$
d_{2}\left(\Phi, F_{a}\right) \leq \frac{d_{2}\left(\Phi, T_{a}[\Phi]\right)}{1-a^{2}}
$$

The characteristic function of the normal distribution $\Phi$ is given by $C_{\Phi}(\xi)=$ $e^{-\frac{1}{2} \xi^{2}}$, so using (18) and the substitution $w=\sqrt{1-a^{2}} \cdot \xi$, we have

$$
\begin{aligned}
& \frac{d_{2}\left(T_{a}[\Phi], \Phi\right)}{1-a^{2}}=\sup _{\xi \neq 0} \frac{\left|C_{T_{a}[\Phi]}(\xi)-C_{\Phi}(\xi)\right|}{\left(1-a^{2}\right) \xi^{2}} \\
= & \sup _{\xi \neq 0} \frac{\mid C_{F}\left(\sqrt{1-a^{2}} \cdot \xi\right) e^{-\frac{1}{2}(a \xi)^{2}}-e^{-\frac{1}{2} \xi^{2} \mid}}{\left(1-a^{2}\right) \xi^{2}} \\
= & \sup _{\xi \neq 0}\left[e^{-\frac{(a \xi)^{2}}{2}} \cdot \frac{\left|C_{F}\left(\sqrt{1-a^{2}} \cdot \xi\right)-e^{-\frac{1}{2}\left(1-a^{2}\right) \xi^{2}}\right|}{\left(1-a^{2}\right) \xi^{2}}\right] \\
= & \sup _{w \neq 0}\left[e^{-\frac{(a w)^{2}}{2\left(1-a^{2}\right)}} \cdot \frac{\left|C_{F}(w)-e^{-\frac{1}{2} w^{2}}\right|}{w^{2}}\right]=\sup _{w \neq 0}\left[e^{-\frac{(a w)^{2}}{2\left(1-a^{2}\right)}} \cdot \frac{\left|C_{F}(w)-C_{\Phi}(w)\right|}{w^{2}}\right] .
\end{aligned}
$$

Combining (24) and (25) we have the result.

We can now give the proofs of the theorems.

Proof of Theorem [1. By Lemma 2 it suffices to show that

$$
\lim _{a \rightarrow 1} \sup _{w \neq 0}\left[e^{-\frac{(a w)^{2}}{2\left(1-a^{2}\right)}} \cdot \frac{\left|C_{F}(w)-C_{\Phi}(w)\right|}{w^{2}}\right]=0 .
$$

Fix $\epsilon>0$. By the assumption (1), $C_{F}$ and $C_{\Phi}$ are twice differentiable, with

$$
C_{F}(0)=C_{\Phi}(0)=1, \quad C_{F}^{\prime}(0)=C_{\Phi}^{\prime}(0)=0, \quad C_{F}^{\prime \prime}(0)=C_{\Phi}^{\prime \prime}(0)=-1
$$

so application of L'Hospital's rule gives

$$
\lim _{w \rightarrow 0} \frac{C_{F}(w)-C_{\Phi}(w)}{w^{2}}=0 .
$$

Therefore we can choose $\delta>0$ so that

$$
|w|<\delta \Rightarrow e^{-\frac{(a w)^{2}}{2\left(1-a^{2}\right)}} \cdot \frac{\left|C_{F}(w)-C_{\Phi}(w)\right|}{w^{2}} \leq \frac{\left|C_{F}(w)-C_{\Phi}(w)\right|}{w^{2}}<\epsilon .
$$


Using the fact that $\left|C_{F}(w)\right|,\left|C_{\Phi}(w)\right| \leq 1$, we have

$$
|w| \geq \delta \Rightarrow e^{-\frac{(a w)^{2}}{2\left(1-a^{2}\right)}} \cdot \frac{\left|C_{F}(w)-C_{\Phi}(w)\right|}{w^{2}} \leq \frac{2}{w^{2}} e^{-\frac{(a w)^{2}}{2\left(1-a^{2}\right)}} \leq \frac{2}{\delta^{2}} e^{-\frac{(a \delta)^{2}}{2\left(1-a^{2}\right)}} .
$$

The right-hand side of the above inequality goes to 0 as $a \rightarrow 1$, hence for $a$ sufficiently close to 1 we have

$$
|w| \geq \delta \Rightarrow e^{-\frac{(a w)^{2}}{2\left(1-a^{2}\right)}} \cdot \frac{\left|C_{F}(w)-C_{\Phi}(w)\right|}{w^{2}}<\epsilon .
$$

From (26), (27) we have that, for $a$ sufficiently close to 1 ,

$$
\sup _{w \neq 0}\left[e^{-\frac{(a w)^{2}}{2\left(1-a^{2}\right)}} \cdot \frac{\left|C_{F}(w)-C_{\Phi}(w)\right|}{w^{2}}\right]<\epsilon
$$

concluding the proof.

Proof of Theorem 2. Assume $s>2$. Using Lemma 2 we have

$$
\begin{aligned}
d_{2}\left(F_{a}, \Phi\right) & \leq \sup _{w \neq 0}\left[e^{-\frac{(a w)^{2}}{2\left(1-a^{2}\right)}} \cdot \frac{\left|C_{F}(w)-C_{\Phi}(w)\right|}{w^{2}}\right] \\
& =\sup _{w \neq 0}\left[e^{-\frac{(a w)^{2}}{2\left(1-a^{2}\right)}} \cdot|w|^{s-2} \cdot \frac{\left|C_{F}(w)-C_{\Phi}(w)\right|}{|w|^{s}}\right] \\
& \leq d_{s}(F, \Phi) \cdot \sup _{w \neq 0}\left[e^{-\frac{(a w)^{2}}{2\left(1-a^{2}\right)}}|w|^{s-2}\right]
\end{aligned}
$$

Computing the supremum on the right-hand side of (28) by elementary calculus we find that it is attained at $w= \pm \frac{\sqrt{(s-2)\left(1-a^{2}\right)}}{a}$, hence

$$
\sup _{w \neq 0}\left[e^{-\frac{(a w)^{2}}{2\left(1-a^{2}\right)}}|w|^{s-2}\right]=\left[\frac{(s-2)\left(1-a^{2}\right)}{e \cdot a^{2}}\right]^{\frac{1}{2}(s-2)},
$$

which gives (14).

\section{References}

[1] J.A. Carrillo, G. Toscani, Contractive probability metrics and asymptotic behavior of dissipative kinetic equations, Riv. Mat. Univ. Parma 6 (2007), 75-198.

[2] K.L. Chung, A Course in Probability Theory, 3rd edition, Academic Press, London, 2001. 
[3] P. Embrechts and M. Maejima. The central limit theorem for summability methods of iid random variables, Z. Wahrsch. Verw. Gebiete 68 (1984), 191-204.

[4] W. Feller, An Introduction to Probability Theory and its Applications, Vol. 2, Wiley, New-York, 1968.

[5] E. Gabetta, G. Toscani and W. Wennberg, Metrics for probability distributions and the trend to equilibrium for solutions of the Boltzmann equation, J. Statist. Phys. 81 (1995), 901-934.

[6] H.U. Gerber, The discounted central limit theorem and its Berry-Esseen analogue, The Annals of Mathematical Statistics 42 (1971), 389-392.

[7] T. Goudon, S. Junca and G. Toscani, Fourier-based distances and BerryEsseen like inequalities for smooth densities, Monatsh. Math 135 (2002), 115-136.

[8] L. Horváth, Approximation for Abel sums of independent, identically distributed random variables, Statistics \& Probability Letters 3 (1985), 221-225.

[9] L. Saulis, D. Deltuviené, The discounted limit theorems, Acta Applicandae Mathematicae 90 (2006), 219-226.

[10] W. Whitt, Stochastic Abelian and Tauberian theorems, Z. Wahrsch. Verw. Gebiete 22 (1972), 251-267. 